\title{
UV Resonance Raman Spectroscopy: A Highly Sensitive, Selective and Fast Technique for Environmental Analysis
}

Kan Xiong*

Broad Institute of MIT and Harvard, Cambridge, USA

*Corresponding author: Kan Xiong, Broad Institute of MIT and Harvard, Cambridge, MA 02142, USA, Tel: +16177147000; E-mail: kanxiong@mit.edu

Rec date: 31 October 2014, Acc date: 4 November 2014, Pub date: 6 November 2014

Copyright: @ 2014 Kan Xiong, This is an open-access article distributed under the terms of the Creative Commons Attribution License, which permits unrestricted use, distribution, and reproduction in any medium, provided the original author and source are credited

Keywords UVRR; Environmental analysis techniques; Raman scattering

\section{Editorial}

For analyzing environmental samples, a highly sensitive, selective and fast technique is desirable. This is due to the fact that [1]. Analytes of interest in the environment are often rare, down to the ppb concentration; [2]. The compositions of environmental samples are often rather complex; [3]. There is an increasing need for real-time analysis, such as detecting explosives in airports or monitoring pollutant in waste water. An incisive technique that meets all these challenges is UV Resonance Raman spectroscopy (UVRR).

\section{Introduction to UVRR}

The Raman Effect is an inelastic scattering process where the excitation electromagnetic field and the molecule exchange a quantum of energy. The difference in energy between the incident light and scattering light corresponds to the Raman active molecular vibrational energy (Figure 1).

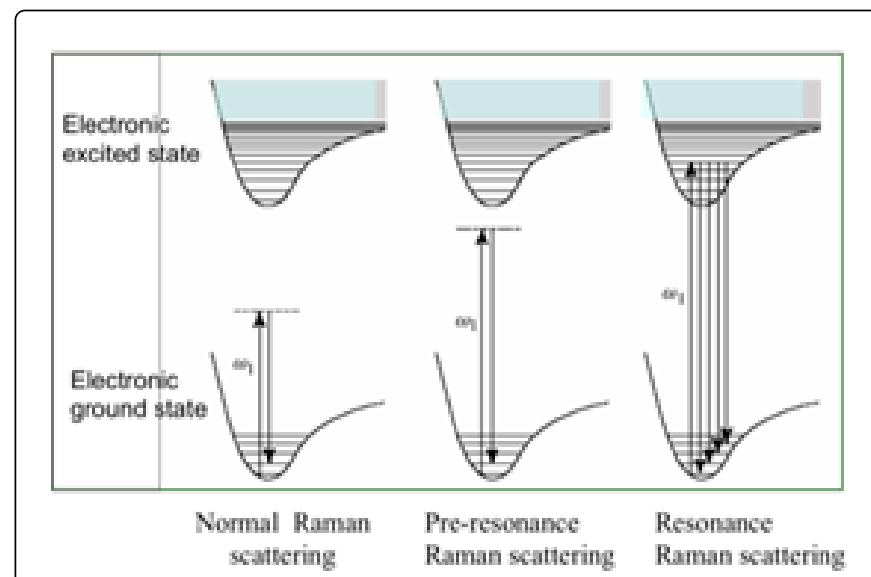

Figure 1: Comparison of normal, pre- resonance and resonance Raman scattering.

The Raman intensities increase approximately with the fourth power of the excitation frequency, $\omega 1$. Resonance Raman scattering occurs when $\omega 1$ equals the electronic transition energy, and the Raman intensities can increase by $10^{6}-10^{8}$-fold, compared to normal Raman scattering. This results in a ultra-high resonance Raman selectivity and sensitivity that makes it a very powerful technique for studying macromolecules; instead of all the sample vibrations contributing with comparable intensities, only a small subset of resonance Raman enhanced vibrations localized around the chromophoric group dominate the spectra. By judiciously tuning the excitation wavelength, we can selectively enhance particular vibrations in particular regions of the macromolecule.

Figure 2 demonstrates the UVRR selectivity for studying the protein myoglobin $(\mathrm{Mb})$. The visible wavelength absorption bands of $\mathrm{Mb}$ result from the in-plane $\pi \rightarrow \pi^{*}$ electronic transitions of its heme group. UVRR excitation of $\mathrm{Mb}$ at $415 \mathrm{~nm}$ in the strong heme Soret absorption band, results in an intense UVRR spectrum which contains only the in-plane heme ring vibrations. In contrast, excitation at $229 \mathrm{~nm}$ within the absorption bands of the tyr and trp aromatic side chains shows UVRR spectra completely dominated by tyr and trp aromatic ring side chain vibrations. Deeper UV excitation at $206.5 \mathrm{~nm}$, within the $\pi \rightarrow \pi^{*}$ transitions of the amide peptide bonds, shows UVRR spectra dominated by the peptide bond amide vibrations. In addition, deep UVRR show increased signal-to-noise ratios due to the lack of significant fluorescence contribution at $\lambda<260 \mathrm{~nm}$. (Asher and Johnson 1984)

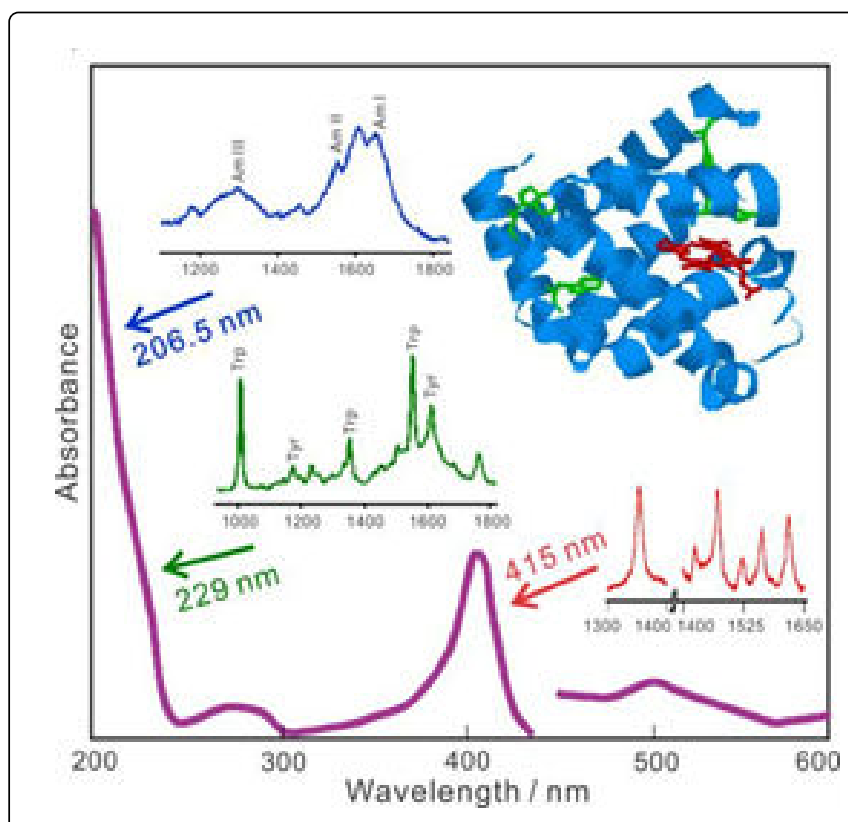

Figure 2: Selectivity of resonance Raman spectral measurements of myoglobin showing the protein absorption spectrum and the different resonance Raman spectra obtained with different excitation wavelengths. Reproduced from ref. (Oladepo, Xiong et al. 2011) 


\section{UVRR Instrumentation}

Figure 3 shows a schematic of a UVRR instrument in brief, a UV laser is utilized to excite within electronic transitions of compound(s) of interest to enhance the vibrations localized around the chromophoric groups. The Raman scattering light is collected and then dispersed by a monochromator onto a CCD camera. The liquid sample is circulated in a free surface, temperature-controlled stream to minimize background. Gas or solid samples can be easily measured too little or no sample preparation is required.

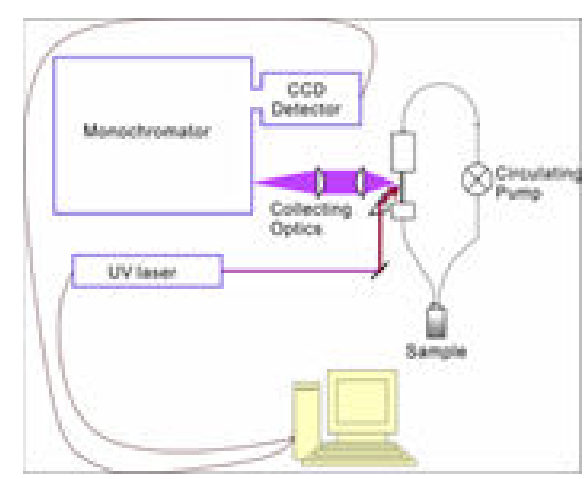

Figure 3: A schematic of a UVRR instrument

\section{UVRR for Environmental Analysis}

Due to its high sensitivity and selectivity, and no requirement for sample preparation, UVRR has been utilized to perform real-time analysis of inorganic compounds (Lanoul, Coleman et al. 2002) and aromatic hydrocarbons including explosives (Johnson and Asher 1984, Tuschel, Mikhonin et al.2010) at ppb concentrations. Figure 4 shows that UVRR allows easy differentiation between structurally similar species down to the $20 \mathrm{ppb}$ level (Johnson and Asher 1984). With rapid advancement of laser technology and Raman spectrometer design, it can be foreseen that in the near future, a portable UVRR device for standoff analysis of environmental samples will become available [1-6].

\section{Conclusion}

With its high sensitivity and selectivity and no requirement for sample preparation, UVRR meets all challenges imposed by modern society for fast and accurate analysis of environmental samples. It is not hard to say that UVRR will play an increasingly significant role in environmental analysis.

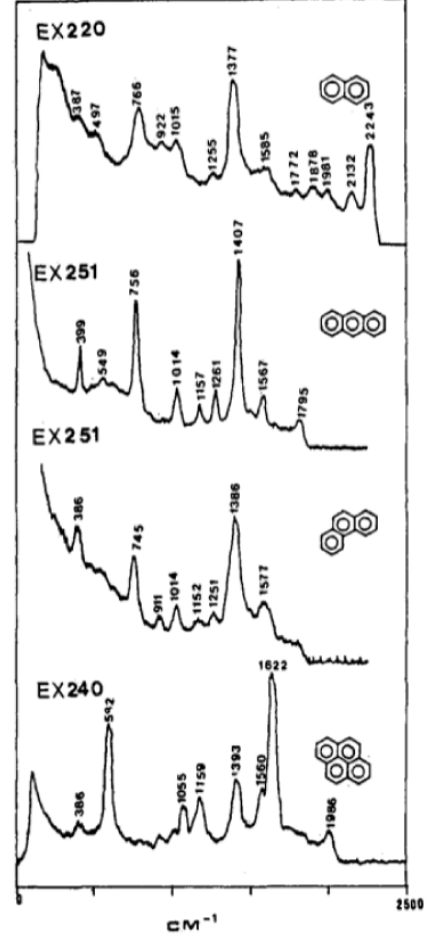

Figure 4: UVRR spectra of naphthalene $(\lambda e x=220 \mathrm{~nm})$, anthracene $(\lambda \mathrm{ex}=251 \mathrm{~nm})$, phenathrene $(\lambda \mathrm{ex}=251 \mathrm{~nm})$ and pyrene $(\lambda \mathrm{ex}=240 \mathrm{~nm})$. Reproduced from ref. (Asher 1984).

\section{References}

1. Asher SA (1984) Ultraviolet Resonance Raman Spectrometry for Detection and Speciation of Trace Polycyclic Aromatic Hydrocarbons. Anal Chem 56: 720-724.

2. Asher SA, Johnson CR (1984) Raman spectroscopy of a coal liquid shows that fluorescence interference is minimized with ultraviolet excitation. Science 225: 311-313.

3. Johnson CR, Asher SA (1984) A new selective technique for characterization of polycyclic aromatic hydrocarbons in complex samples: UV resonance Raman spectrometry of coal liquids. Anal Chem 56: 2258-2261.

4. Lanoul A, Coleman T, Asher SA (2002) UV resonance raman spectroscopic detection of nitrate and nitrite in wastewater treatment processes. Anal Chem 74: 1458-1461.

5. Oladepo SA, Xiong K, Hong Z, Asher SA (2011) Elucidating Peptide and Protein Structure and Dynamics: UV Resonance Raman Spectroscopy. J PhysChemLett 2: 334-344.

6. Tuschel DD, Mikhonin AV, Lemoff BE, Asher SA (2010) Deep ultraviolet resonance Raman excitation enables explosives detection. Appl Spectrosc 64: 425-432. 